\title{
CHARACTERIZATION OF COLLOIDAL SYSTEMS IN A STANDALONE PZT- GLASS CAPILLARY MICROFLUIDIC ULTRASONIC TWEEZER
}

\author{
M.K. Araz ${ }^{*}$ and A. Lal \\ SonicMEMS Laboratory, School of Electrical and Computer Engineering, \\ Cornell University, Ithaca, New York, USA
}

\begin{abstract}
This paper presents an acoustic PZT-glass capillary actuator mechanism for the characterization of colloidal systems. This actuator enables monitoring of the cumulative effect of the nanoscale force interactions between many individual nanoparticles at the millimeter scale which can be tracked by conventional optics. Since the actuation is through acoustical radiation and streaming forces, optical, dielectric or magnetic properties of the materials do not interfere with the actuation method, enabling accuracy in the measurements with applicability to a wide range of samples with different ionic concentrations. In this paper we show that the effect of the ionic charges on silica beads can be measured by collective squeezing and expansion of bead collections.
\end{abstract}

\section{INTRODUCTION}

Colloid characterization is used in many industrial applications such as pharmaceutical products, paints, inks, printing, ceramic casting, paper coating, solvent recovery, water purification, food industry, waste water treatment and disposal of radioactive waste [1]. Surface Plasmon Resonance of colloidal systems also plays a crucial role in biosensors at microscale. While in some applications long term stability of the colloidal system is the aim, in some cases destruction of the colloidal system is desired. Colloidal systems are stable due to counterbalancing of attractive and repulsive interactions present in the system such as; electrostatic, long range and retarded van der Waals, hydrodynamic and steric interactions. In most cases, electrostatic repulsion is the dominant interaction mechanism determining colloidal stability. Due to the many parameters affecting the surface charge and the surrounding ionic diffuse layer, and the shortcomings of the current theoretical models, characterization of the stability of the colloidal systems is mostly done experimentally in manufacturing.

In this paper a PZT(Lead Zirconate Titanate) driven glass capillary microfluidic actuator is used for the characterization of the colloidal systems. A picture of the device is shown in Figure 1. As a novel advantage, actuator mechanism enables monitoring of the cumulative effect of the nanoscale force interactions between many individual nanoparticles at the millimeters scale which can be tracked by simple optics. Acoustic radiation forces generated inside the microfluidic capillary enable collection of the colloidal structures at desired locations [2-3]. With the release of the acoustic forces particles collectively diffuse back to the medium. Through the monitoring of the collective diffusion behavior, the stability characteristic of the colloidal system is obtained. Since the actuation is done through acoustical forces, optical, dielectric or magnetic properties of the materials do not interfere with the actuation method, enabling accuracy in the measurements with applicability to a wide range of samples. There is no need of electrical contacts within the fluid inner-core, eliminating the need of calibration of electrode-fluid contacts for various samples (ionic, polar, non-polar, acidic, etc.), prevents bubble formation and makes the actuator mechanism usable for delicate biological samples and plasmonic biodetection.

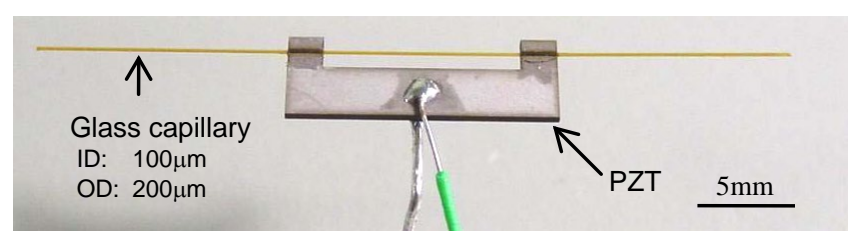

Figure 1. Picture of the PZT-glass capillary actuator. Capillary inner diameter is $100 \mu \mathrm{m}$ and outer diameter is $200 \mu \mathrm{m}$. Outside of the capillary is coated with polyimide enabling the flexibility of the glass capillary.

\section{COLLOIDAL BEHAVIOR AND THE SURFACE CHARGE}

The term colloid can be defined as a system of two substances, in which one substance is distributed evenly in a discrete phase throughout the second substance of continuous phase. The dispersed materials and the continuous medium may be in gaseous liquid or solid phases. In this study, point of interest is given to the colloidal particles suspended in liquids, which is the most common type of colloidal system, and is relevant for micro and nanofluidic systems.

\section{Diffusion}

Microscopic particles suspended in liquids exhibit Brownian motion due to the random momentum transfer of the colliding molecules of the medium they are suspended in. This random motion enables single particles to move, and in case of many particles leads to diffusion. Laws of diffusion were first formulated by Fick [4] and for $N_{O}$ number of non interacting colloidal particles introduced to a medium at zero time and location, $t=0, z=0$, the particle distribution, $n(z, t)$, is given by

$$
n(z, t)=\frac{N_{0}}{\sqrt{4 \pi D t}} e^{-\left(z-z_{0}\right)^{2} / 4 D t}
$$

where $D$ is the diffusion constant. Theoretically derived by Einstein [5], $D$ is given by

$$
D=\frac{k T}{6 \pi \eta a}
$$

where $k$ is the Boltzmann constant, $T$ is the temperature in Kelvin, $\eta$ is the viscosity of the medium and $a$ is the particle radius. This expression is valid for low-concentration of solute colloids. For a particle having size of $500 \mathrm{~nm}$, suspended in water, at room temperature, the diffusion constant is expected to be around 0.85 $\mu \mathrm{m}^{2} / \mathrm{s}$ which is in the order of experimentally obtained values given in the literature [6].

Equations 1 and 2 explain diffusion phenomena for noninteracting particles. However, this is not a very common scenario as usually particles undergo hydrodynamic, Van der Waals and electrostatic interactions.

\section{Surface Charge and the Zeta potential}

Many particles bear surface charges when they are suspended inside a fluidic medium. Usually this phenomenon stems from capturing of ions present inside the fluid, or losing some surface ions present on the particle or both. Due to this ionic exchange, as shown in Figure 2, an electrical double layer forms in the fluid particle boundary region. Inner ions in the double layer are attached to the particle surface and move with it, and they form the 


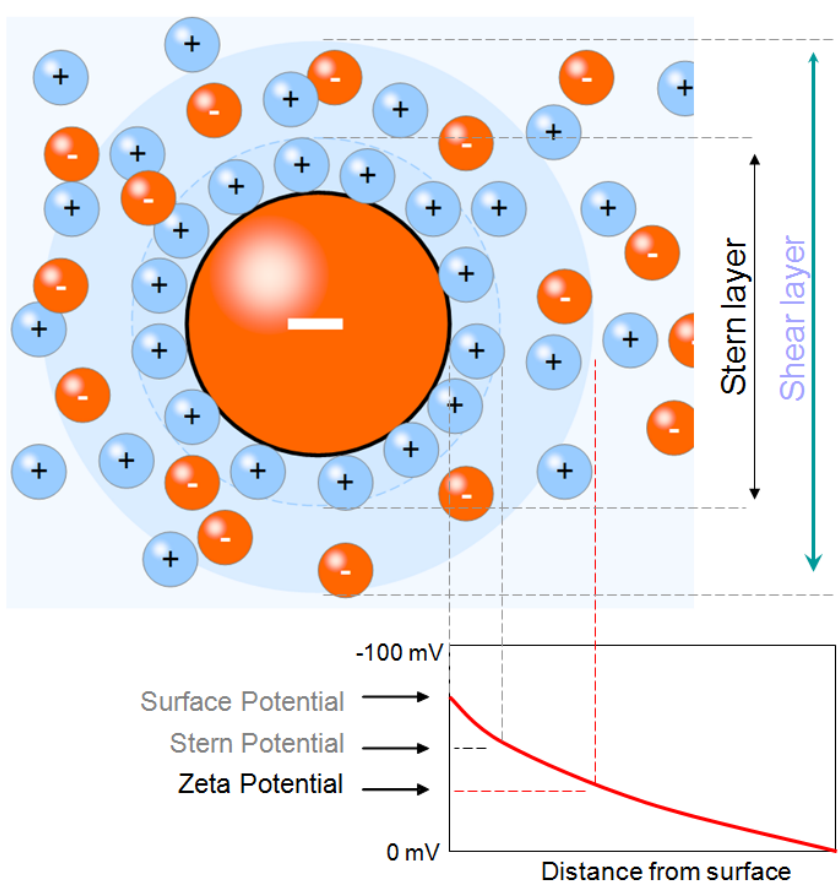

Figure 2. Electrostatic behavior of a nanoparticle in an ionic fluid is determined by the ionic double layer and the surrounding shear layer. Zeta potential value at the end of the shear layer is the only measurable potential defining the characteristics. [8]

Stern layer [7]. Outside the Stern layer, remaining counter ions form a diffuse region which defines the shear layer in which particle moves with respect to the stationary fluid molecules. The electric potential at this shear layer is called the zeta potential $(\zeta)$ which defines the electrokinetic properties of the particle. Being the only measurable quantity related to the charge information of the particle inside the fluid, zeta potential is used in place of surface potential in analytical calculations. Zeta potential can be obtained from the electrophoretic mobility of the particles by formula

$$
\zeta=\frac{3 \eta v}{2 \varepsilon E f(\kappa a)}
$$

where $\eta$ is the viscosity, $v$ is the particle velocity, $\varepsilon$ is the dielectric constant of the medium, $E$ is the electric field, and $f(\kappa a)$ is a constant number, and $a$ the radius of the particle; in this case $f(\kappa a)$ is around 1.5 [7]. $\kappa^{-1}$ is the Debye (diffuse) layer thickness and is related to the ionic strength of the solution. In our experiments Zetasizer Nano Z series by Malvern Instruments is used to characterize zeta potential of the particle samples.

The interaction potential between two colloidal particles is modeled by DLVO (Derjaguin, Landau, Verwey and Overbeek) theory, which is an approximation obtained by taking attractive van der Waals interactions and double layer electrostatic repulsion into consideration and given by

$$
U=-\frac{A_{12} a}{12 h}+\pi \varepsilon \zeta^{2} a e^{-\kappa h}
$$

where the first term represents the attractive van der Waals interaction and the second term electrostatic repulsion. In the formula, $A_{12}$ is the Hamaker constant and $h$ is the separation between two particles [7].

While this formula has been tested for two particle systems in well defined media extensively, practical applicability to experiments is mostly limited. This is first due to existence of many particles in a colloidal system and secondly many parameters such as zeta potential and the Debye length depend strongly in the
$\mathrm{pH}$ and the ionic concentration of the media as well as the media type. In this regard, zeta potential becomes one of the key parameters defining colloidal stability in experimental studies. By changing the $\mathrm{pH}$ of the medium, colloidal stability can be altered drastically due to the change in the zeta potential. Colloidal particles which have zeta potential value of between $\pm 30 \mathrm{mV}$ tend to aggregate as the electrostatic repulsion cannot balance the attractive forces.

Because of the challenges mentioned above, experimental characterization of colloidal systems is an inevitable step in the product development or research. Characterization can be costly due to the long time span of experiments and the requirement of bulky and expensive equipment. For example, from early development to FDA approval timeline of a pharmaceutical product can take up to 10-20 years and cost hundreds of millions of dollars. While the significant portion of this effort goes into clinical trials, manufacturing of the drugs with proper delivery methods add also considerably to the cost and time spent.

\section{EXPERIMENTAL RESULTS}

\section{Fast collective diffusion behavior}

As shown in Figure 1, microfluidic actuator consists of a polyimide coated fused silica capillary (ID: $100 \mu \mathrm{m}$, OD: $100 \mu \mathrm{m}$, PolyMicro Technologies) which is adhesively bonded to a laser cut PZT-4 plate. PZT plate is driven at one of the resonance frequencies of the flexural modes of the cylindrical capillary in the $30 \mathrm{kHz}-2 \mathrm{MHz}$ regime. Flexural modes of the capillary generate sub-wavelength stationary acoustic waves inside the capillary at vibration maxima. As shown in Figure 3, these stationary acoustic waves lead to acoustic radiation forces at the center of vibration maxima [3]. These acoustic forces act as periodic planar centrifugal fields collecting colloidal particles at preferred locations. Collection of $3 \mu \mathrm{m}$ size particles shown in Figure 3 is in agreement with the theoretically predicted force field. Details of the fluid-structure coupling and the analytical model will be presented in a future reference (also given in [3]).

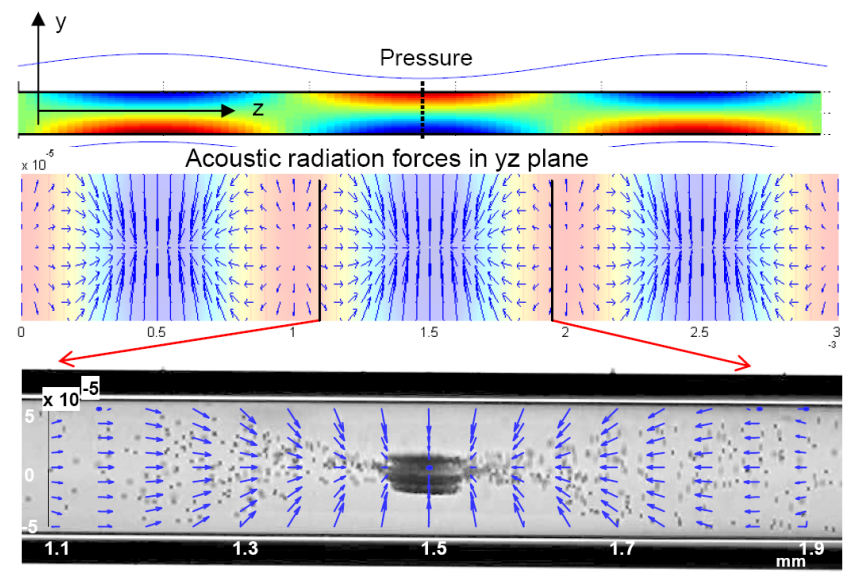

Figure 3. Flexural vibrations generate sub-wavelength stationary acoustic fields (top) which lead to acoustic radiation forces at the vibration maxima (middle). Microscope image shows theoretical forces and the collection of $3 \mu \mathrm{m}$ beads suspended in the fluid enclosed in the capillary at a vibration maxima (bottom).

Size of the colloidal nanoparticles (Kisker Scientific, Bangs Laboratories) used in the experiments presented here are $500 \mathrm{~nm}$ silica nanoparticles dispersed in de-ionized water. Since the glass capillary is transparent, collection and the dispersion of the regular nanoparticles can be monitored through light scattering. Experimental setup sketched in Figure 4 eliminates the necessity of 


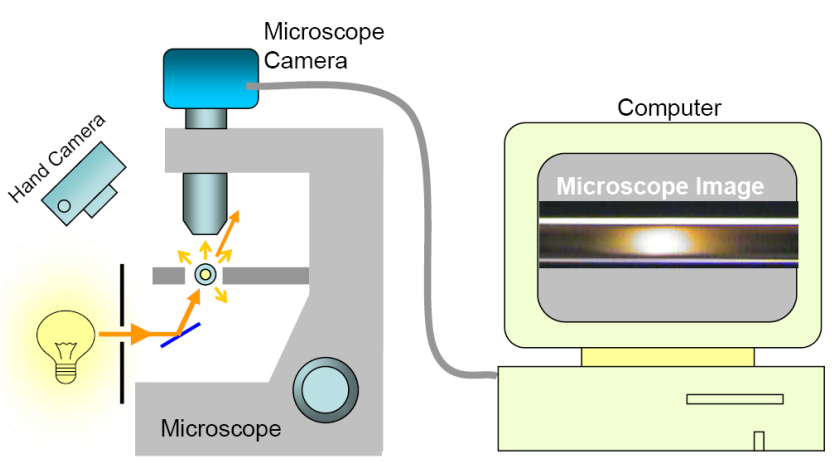

Figure 4. Schematics showing the experimental setup related to the imaging. Part of the light scattered from the colloidal particles inside the capillary is collected at the microscope objective and transferred to the computer through the microscope digital camera.

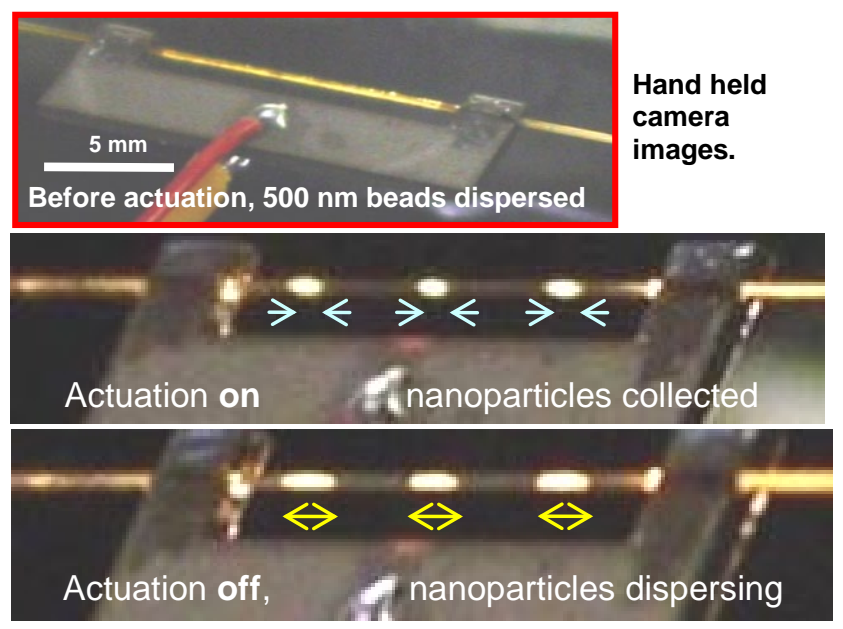

Figure 5. Hand held camera images of the actuator with 500nm silica beads suspended in DI water. Locations where there are silica nanobeads shine due to the scattering of the light coming from the bottom. Top: before the actuation, beads are dispersed. Middle: Nanoparticles are collected. Bottom: Actuation is oft and the collective diffusion of the nanoparticles is observed (650 times faster than expected diffusion rate for individual beads).

fluorescent labels for the monitoring of the colloidal particles. Hand held camera images displayed in Figure 5 show that, results are visible to eye without any magnification. Before the actuation, a continuous scattering through the capillary is observed. After actuation is turned on, light is scattered only at certain locations where silica particles are focused.

After the collection of particles, with the turning off the actuation, particles tend to diffuse back to their original dispersed state over time. This expansion can be seen in hand held camera images in Figure 5 and timed microscope images shown in Figure 6. From the series of images shown in Figure 6, it is possible to correlate the scattered light intensity with the particle number density. Since the capillary inner diameter is small, particle concentration assumed to depend linearly on the light intensity.

The graph displayed on top in Figure 7 shows the normalized particle volume fraction obtained from intensity distributions. As the particles disperse with time, spatial particle volume fraction decreases.

Here, the observed collective diffusion constant of the collected $500 \mathrm{~nm}$ silica particles $\left(550 \mu \mathrm{m}^{2} / \mathrm{s}\right)$ is found to be about 650 times

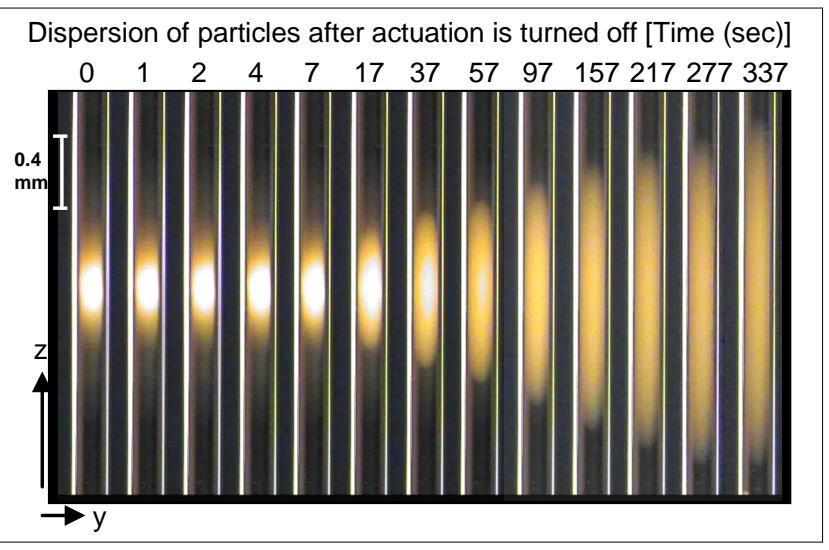

Figure 6. Following the collection of particles when the actuation is turned off, particles spread back inside the capillary. Particle size density or volume fraction is correlated to the intensity of the light scattered by the colloidal silica nanobeads.
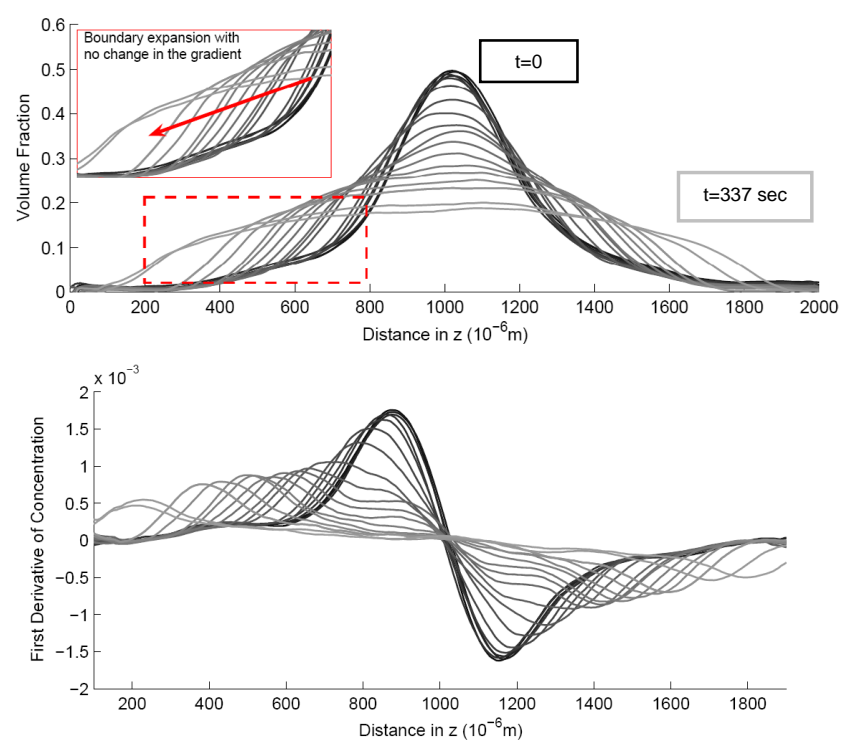

Figure 7. Top: Change in the volume fraction over time after the release of the acoustic force. Boundary moves 650 times faster than the expected diffusion constant of the 500nm nanoparrticles. Bottom: Derivative of the concentration profile; peaks indicate the location of the boundary at a specific time.

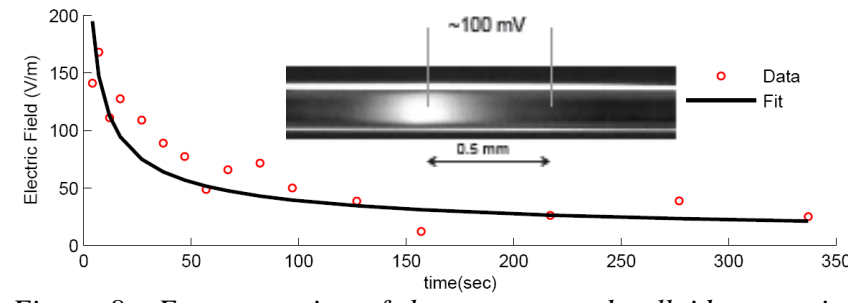

Figure 8. Fast expansion of the concentrated colloid system is associated with the self generated macroscopic electric field. From the velocity of the moving boundary, magnitude of the electric field, which gives the charged particles their momentum, can be obtained.

higher than the expected diffusion constant of individual particles $\left(0.85 \mu \mathrm{m}^{2} / \mathrm{s}\right)$, which gives a measure of the collective diffusion behavior of the colloidal system. This fast collective diffusion behavior cannot be explained by regular diffusion or built up of 
osmotic pressure due to the particle collection, and can only be attributed to electrostatic effects generated due to the collection particles in a small volume. Through the monitoring of the moving boundary in the volume fraction plots in Figure 7, which are obtained from scattered light intensity, built in electric field values causing this fast collective diffusion behavior are calculated. Velocity of the expansion at the beginning of radiation force removal is in the order of tens of $\mu \mathrm{m} / \mathrm{s}$ which correspond to macroscopic electric field values around $200 \mathrm{~V} / \mathrm{m}$. Electric field values calculated from experimental data over the course of expansion is given in Figure 8. This high electric field values are reached because of the fact that all particles, initially dispersed inside the capillary over a region of few millimeters, are confined to a range of $400 \mu \mathrm{m}$. This collection raises the particle volume fraction from 0.11 to about 0.6 which is very close to the close packed crystal structure in which all particles are packed being in touch with their closest neighboring particles. In this regard the electrical potential built up can be attributed with the sedimentation potential obtained during the gravitational or centrifugal sedimentation experiments. Here since the particles are confined to a smaller microscopic region results are amplified.

\section{Acoustic focusing at various $\mathrm{pH}$ values}

As mentioned above, zeta potential depends on the $\mathrm{pH}$ of the solution. Figure 9 shows the actual zeta potential measurements of $300 \mathrm{~nm}$ and $500 \mathrm{~nm}$ silica nanoparticles in solutions having various $\mathrm{pH}$ values. Measurements are performed in a Zetasizer Nano by Malvern Instruments. It is observed that as the acidity of the solution increases (decreasing $\mathrm{pH}$ ), zeta potential around the particle starts to decrease. This is due to the collection of $\mathrm{H}^{+}$ions around the negatively charged particles. At $\mathrm{pH}$ levels below 4, concentration of $\mathrm{H}^{+}$ions are high enough to even reverse the polarity of the zeta potential at the diffuse layer. When zeta potential is between $30 \mathrm{mV}$ and $-30 \mathrm{mV}$, it has been known that electrostatic repulsion cannot balance the attractive forces and flocculation is expected. In this case, some nanoparticles start to stick to each other and the effective particle size gets higher. Particle size characterization experiments reveal that, for instance at low $\mathrm{pH}$ values, rather than a single peak at $500 \mathrm{~nm}$, a broader spectrum with peaks at higher values such as $700 \mathrm{~nm}$ or $1200 \mathrm{~nm}$ are observed. This change can also be observed in PZT-glass capillary actuator without any need of particle size or zeta potential measurement. As shown in Figure 10, collection of $500 \mathrm{~nm}$ silica particles at different $\mathrm{pH}$ values give different particle distributions due to charge effects. In the top image, at $\mathrm{pH} \mathrm{7,500} \mathrm{nm}$ silica particles collect in a cloudy pattern where as at $\mathrm{pH} 3$, there are narrower and denser collections at the center. This is due to the fact that as particles aggregate, they feel a bigger radiation force which leads to a higher collection efficiency. Hence, this device enables the monitoring of the diminished colloidal stability.

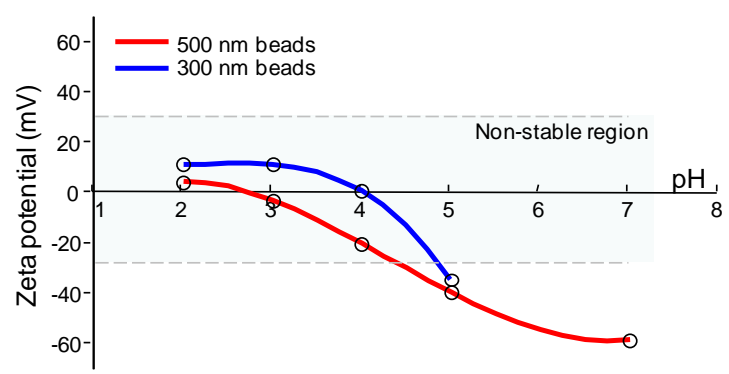

Figure 9. Zeta potential measurements of $300 \mathrm{~nm}$ and $500 \mathrm{~nm}$ silica beads suspended in aqueous solution of different $\mathrm{pH}$ levels.

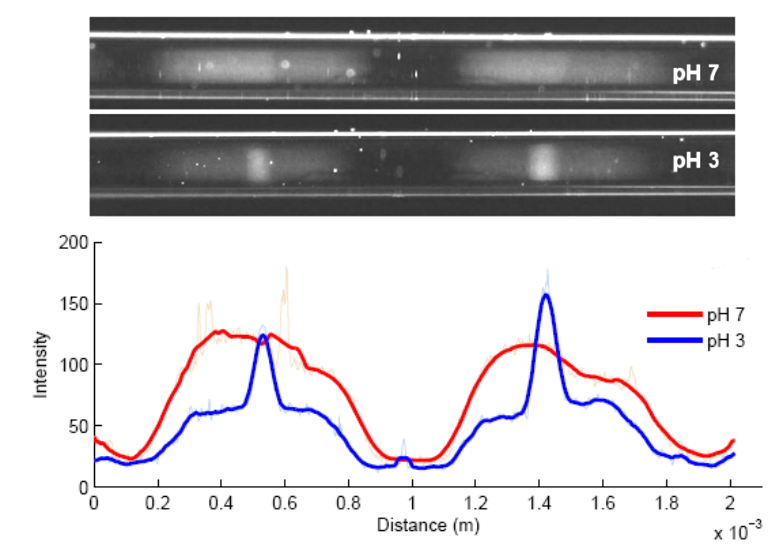

Figure 10. Collection of $500 \mathrm{~nm}$ nanoparticles. The top image shows the particles suspended in a pH 7 solution. The middle image shows particles suspended in a $\mathrm{pH} 3$ solution. Plotted at the bottom is the intensity of the scattered light measured at the central axis along the capillary.

\section{CONCLUSIONS}

A possible use of PZT-glass capillary actuator for the characterization of the colloidal systems is introduced. The actuator presented here may replace bulky centrifugal systems or eliminate time consuming sedimentation experiments. In addition results can be obtained very fast with low sample volume requirement ( 0.6 microliters). Since the amount of power consumed by the actuator is very low, portable battery operated hand held devices are also possible. Even the internal diameter of the capillary is 100 micrometers; results are observable to the naked eye and can be monitored in detail with a regular hand held digital camera or a document scanner. The capillary used is the standard fused silica capillary and can be integrated with wide range of other larger or same size bio analytical tools such as HPLC or other microfluidic systems. Device can be used to monitor behavior of the colloids at various $\mathrm{pH}$ values. In the analysis of the colloidal systems, since the theory can deviate from experimental results especially in high salt concentrations or acidity, direct experimental observation is always performed in product development. In this regard, PZT-glass capillary actuator may be a fast, reliable and cost efficient solution for the characterization of colloidal stability problem.

\section{REFERENCES}

[1] D.H. Everett, Basic Principles of Colloid Science, Royal Society of Chemistry, 1988

[2] C.H. Lee, A. Lal, Low-Voltage High-Speed Ultrasonic Chromatography for Microfluidic Assays, Hilton Head, 2002

[3] M.K. Araz, Generation of Sub-wavelength Acoustic Stationary Waves in Microfluidic Platforms: Theory and Applications to..., Ph.D. Thesis, Cornell University, 2010.

[4] A. Fick. On liquid diffusion. Phil. Mag., 10:30, 1855.

[5] A. Einstein. Investigations on the Theory of the Brownian Movement. Dover Publications, 1956.

[6] B. Rieger, H.R.C. Dietrich, L.R. van den Doel, and L.J. van Vliet. Diffusion of Microspheres in Sealed and Open Microarrays. Microscopy Res and Tech, 65:218-225, 2004.

[7] I.D. Morrison and S. Ross. Colloidal Dispersions: Suspensions, Emulsions and Foams. Wiley, 2002.

[8] http://www.malvern.com/

\section{CONTACT}

*M.K. Araz, tel: +1-607-255-1815; mka22@cornell.edu 